\title{
Micro-Systems Production: A Promising New Technique with Low Energy Consumption
}

\author{
A. DALmoro ${ }^{1}$, A. A. BARBA ${ }^{2}$, M. D'AMORE ${ }^{2}$, G. LAMBerti $^{1}$ \\ ${ }^{1}$ Dipartimento di Ingegneria Chimica e Alimentare, Università di Salerno, Fisciano, Italia \\ 2 Dipartimento di Scienze Farmaceutiche, Università di Salerno, Fisciano, Italia \\ E-mails: adalmoro@unisa.it (A. Dalmoro), aabarba@unisa.it (A.A. Barba), \\ mdamore@unisa.it (M. d'Amore), glamberti@unisa.it (G. Lamberti)
}

Sci Pharm. 2010; 78: 670

doi:10.3797/scipharm.cespt.8.PMS43

Nowadays, a considerable range of industrial applications (pharmaceutics, cosmetics, foods agriculture products) relies on microencapsulation of solids or liquids by polymer coating and entrapment into polymer matrices.

Production of these micro-systems doubtless represents one of the most important improvements in the development of functional and therapeutic products belonging to the industrial fields above reported.

Generally, microencapsulation is widely used in pharmaceutical and nutraceutical fields, mainly to improve stability or to modify active principles release. Smart micro-systems release active molecules in fuction of external stimuli $(\mathrm{pH}$, temperature). Preparation of the microparticles is achieved by both physico-chemical and mechanical processes. Feasibility studies about a process of microencapsulation are achieved by manual procedures in a labscale. The path toward commercialization of micro-particles needs plants or automated equipments on lab- or pilot-scale first, eventually on industrial scale. However, the state-of-art about microencapsulation techniques displays several processes based on manual operations that often don't find an applicative realization in industrial field. Moreover, equipments reported in literature, especially in lab-scale systems, are not versatile devices and are penalized by a high consumption of resources. This work is focused on the development of techniques able to overcome such limitations. A promising new technique with

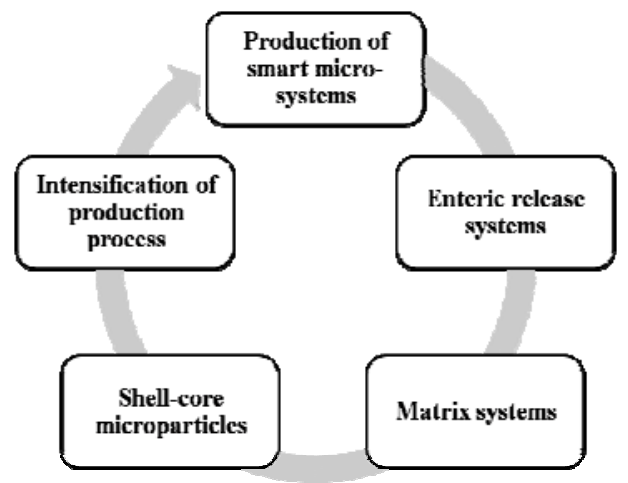
low energy consumption consists in the coupling between ultrasonic atomization and microwave drying to produce micro-systems through an intensified process. The variation of energy/frequency of ultrasounds gives the dimensional distribution more suitable for the final applications. The microwave drying modifies materials structure giving unique properties to the micro-particles. 